\title{
EQUIVARIANT DYNAMICAL SYSTEMS
}

\author{
BY MIKE FIELD ${ }^{1}$
}

Communicated by Stephen Smale, May 11, 1970

In this note we consider equivariant vector fields and diffeomorphisms and present results which generalise some well-known theorems of the theory of dynamical systems as developed by Smale and others. The main result is a generalisation of the Kupka-Smale density theorem. Proofs will be given elsewhere; the note is a summary of the author's Ph.D. thesis, done at the University of Warwick.

For a survey of dynamical systems theory, see [1]; for elementary facts about equivariant vector fields, see [2].

$M$ will always denote a compact $C^{\infty}$ manifold, without boundary, and $G$ a compact Lie group acting differentiably on $M$. Let $G V B(M)$ and $G F B(M)$ respectively denote the categories of $C^{\infty} G$-vector bundles and $C^{\infty} G$-fiber bundles over $M$; we assume paracompact fiber.

Thus $T M \in G V B(M)$ in a natural way. For $E \in G V B(M)$ or $G F B(M)$ we may consider $C_{G}^{r}(E)=\left\{X \in C^{r}(E): g X g^{-1}(x)=X(x), g \in G, x \in M\right\}$ : The space of equivariant sections of $E$. It is well known that for $E \in G V B(M), C_{G}^{r}(E)$ is a Banach splitting subspace of $C^{r}(E)$, with respect to the $C^{r}$ topology on $C^{r}(E)$.

As a straightforward generalisation of the proof given for $G=\mathrm{id}$ in [3], we have:

Theorem 1. If $E \in G F B(M)$, then $C_{G}^{r}(E) \subset C^{r}(E)$, as a closed $C^{\infty}$ Banach submanifold, in a natural unique way.

Corollary 1.1. $\operatorname{Diff}_{G}^{r}(M) \subset C^{r}(M, M), r \geqq 1$, as a $C^{\infty}$ submanifold. Here $\operatorname{Diff}_{G}^{r}(M)$ denotes the set of $C^{r}$ equivariant diffeomorphisms of $M$, with the $C^{r}$ topology.

If $X \in C_{G}^{r}(T M)$ and $X(x)=0_{x}$, then $X(g x)=0_{g x}, g \in G$, and $G(x)$ is a singular set for $X$. Similarly for $f \in \operatorname{Diff}_{G}^{r}(M)$, with $f x=x$.

Let $q$ be a closed orbit of $X \in C_{G}^{r}(T M)$. We define $G_{q}$, the "isotropy group of $q$ ", by:

AMS 1969 subject classifications. Primary 3465; Secondary 5731, 5747.

Key words and phrases. Equivariant vector fields, equivariant diffeomorphisms, compact Lie group action, manifolds of equivariant maps, normal hyperbolicity, genericity, transversality, Kupka-Smale density theorem, Hartman's theorem, Morse-Smale system, Anosov diffeomorphism, structural stability.

${ }^{1}$ Research done whilst the author was receiving an S.R.C. grant. 


$$
G_{q}=\{g \in G: g(q)=q\} .
$$

Then $G_{q}$ is a closed subgroup of $G$ and, if $x \in q, G_{x}$ (the isotropy group of $x$ ) is contained in $G_{q}$ as a closed normal subgroup.

We may easily show that there are two types of orbits:

1. $G_{q} / G_{x} \cong C^{k}$, where $C^{k}$ is the cyclic group of order $k$.

2. $G_{q} / G_{x} \cong S^{1}$, which implies, in particular, that $G(q)=G(x)$ and that $q$ is a $C^{\infty}$ closed orbit of $X$.

We now recall a definition of [4], adapted here to the equivariant situation:

Definition 1. Let $V$ be a $C^{1}$ compact submanifold of $M$ and let $f \in \operatorname{Diff}_{G}^{\gamma}(M)$. We suppose that $V$ is both $G$-invariant and $f$-invariant and that $M$ is a Riemannian manifold.

We say $f$ is " $G, r$-normally hyperbolic on $V$ " iff the tangent bundle of $M$, restricted to $V$, splits into three continuous subbundles:

$$
T_{V} M=T V \oplus N^{s} \oplus N^{u},
$$

invariant by the differential of $f, T f$, and s.t.:

1. $\sup _{x \in V}\left\|T f^{k} \mid N_{x}^{s}\right\|<\inf _{x \in V}\left(m\left(T f^{k} \mid T_{x} V\right)\right) \quad 1 \leqq k \leqq r$, and $T f$ contracts $N^{s}$.

2. $\inf _{x \in V}\left(m\left(T f^{k} \mid N_{x}^{u}\right)\right)>\sup _{x \in V}\left\|T f^{k} \mid T_{x} V\right\| 1 \leqq k \leqq r$, and $T f$ expands $N^{u}$.

Here $m(A)=\inf \{|A x|:|x|=1\}$.

Essentially the normal behaviour of $T f$ is hyperbolic and it dominates the tangent to $V$ behaviour.

As an easy consequence of the definition, we may assume that $M$ is $G$-Riemannian and that the splitting induced by $T f$ is $G$-invariant.

For $C^{r}$ equivariant flows $\left\{f^{t}\right\}$ we have a similar definition with $f^{t}$, for some $t>0$, replacing $f$ in the above.

DEFINITION 2.

Case A: If $G(x)$ is a singular set for $X \in C_{G}^{\gamma}(T M)$, we say $G(x)$ is a 1-generic singular set for $X$ iff $G(x)$ is $G, r$-normally hyperbolic for $X$ (i.e. the flow of $X$ ). Similarly for diffeomorphisms.

Case B: If $q$ is a closed orbit, we say $X$ is 2-generic on $G(q)$ iff $G(q)$ is $G, r$-normally hyperbolic for $X$-we note the difference between Type 1 and Type 2 closed orbits.

We define a generalised Poincaré map, for a closed orbit of Type 1, by taking a $C^{r} G$ normal bundle of $G(q)$, restricting to $G(x), x \in q$, and projecting into $M$ using the exponential map to define a transverse section to the orbit through $x$, the Poincare map so defined is $C^{c}$ equivariant with a fixed set $G(x)$, denote the map by $f_{X}$.

Then $f_{X}$ is 1 -generic on $G(x)$ iff $X$ is 2-generic on $G(q)$. 
If $H \subset G$ is a subgroup, we let $N(H)$ denote the normaliser of $H$ in $G$.

If, in addition in Case A, $\operatorname{dim}\left(N\left(G_{x}\right)\right)=\operatorname{dim} G_{x}$, we say that $G(x)$ is a $1^{*}$-generic fixed set for $X$, or for $f$, if $f$ is a diffeomorphism.

If in Case $\mathrm{B}, q$ is an orbit of Type 1 and $f_{X}$ is $1^{*}$-generic, we say that $G(q)$ is a $2 *$-generic set for $X$. If $q$ is an orbit of Type 2, we say it is $2 *$-generic iff rank $\left(N\left(G_{x}\right) / G_{x}\right)=1$.

These additional conditions essentially imply that under peturbation closed orbits are stable, i.e. do not perturb into noncompact orbits.

THEOREM 2. With the above notation, the set of equivariant vector fields, satisfying the property that all singular points are $1^{*}$-generic and all closed orbits are $2^{*}$-generic, is a residual subset of $C_{G}^{r}(T M)$.

The proof of this result is long and we do not attempt a summary here. We have a similar theorem for diffeomorphisms.

If $q$ is a 2-generic closed orbit of $X$, we have global stable and unstable manifolds for $G(q)$; in fact we have:

TheOREM 3. If $\bar{N}^{s}$ is a $C^{r} G$ vector bundle approximation to $N^{s}$ (see Definition 1) then there exists a $C^{r}$ injective equivariant immersion $I: \bar{N}^{s} \rightarrow M$, s.t. $: I\left(\bar{N}^{s}\right)=W^{s}(G(q))$ and also $I\left(\bar{N}^{s} \mid q\right)=W^{s}(q)$.

Similarly for the unstable manifold of $G(q)$ and for 1-generic singular sets.

REMARK.

1. Such $G$-vector bundle approximations can be shown to exist.

2. $W^{*}(G(q))$ is characterised by:

$$
W^{*}(G(q))=\left\{z \in M: F_{t} z \rightarrow G(q) \quad \text { as } t \rightarrow \infty\right\} ;
$$

similarly for the other sets.

If $W \subset M$, and $H \subset G$ as a closed subgroup, we set $W_{H}$ $=\left\{z \in W: G_{z}=H\right\}$.

Let $G(p)$ and $G(q)$ be two generic critical sets of $X \in C_{G}^{r}(T M)$, we say $W^{s}(G(p))$ meets $W^{u}(G(q))$ "weak $G$ transversally at $y \in M^{\prime}$ " iff:

$$
\left(W^{s}(G(p))\right)_{G_{y} \AA_{y}}\left(W^{u}(G(q))\right)_{G_{y}} \subset M_{G_{y}},
$$

i.e. we require that the intersection is transversal at $y$ in $M_{G_{y}}$.

We say $W^{s}(G(p))$ meets $W^{u}(G(q))$ weak $G$ transversally iff all points of intersection are weak $G$ transversal.

We say $X \in C_{G}^{\gamma}(T M)$ is 3-generic iff $X$ is $1^{*}$ and $2^{*}$ generic and the 
stable and unstable manifolds of generic critical elements of $X$ are weak $G$ transversal. We have:

TheOREM 4. $C_{G}^{r}(T M)$ contains a residual subset of 3-generic vector fields.

Whilst weak $G$ transversality is the strongest transversality condition one can reasonably ask for in this context, nevertheless difficulties arise as, even if $W \subset M$ is compact $W_{H} \subset M$, for $H \subset G$, will, in general, be noncompact.

As an easy consequence of work in [4], we have:

Theorem 5 (Generalised Equivariant Hartman's Theorem). If $p$ is a generic critical element of $X \in C_{G}^{r}(T M)$, we have an equivariant conjugacy $h$ between $\left\{F_{t}^{X}\right\}$ and $\left\{N F_{t}^{X}\right\}$ in a nbd. of $G(p)$. Here $\left\{F_{l}^{X}\right\}$ denotes the flow of $X$, and $N F_{t}^{X}=T F_{t}^{X} \mid\left(N^{s} \oplus N^{u}\right)$. $h$ is a homeomorphism.

Similarly for diffeomorphisms.

We end with a brief consideration of some rather more specific problems.

If $f \in \operatorname{Diff}_{G}^{r}(M)$, we say $f$ is a "G-Anosov map" iff:

$$
f_{*}: C_{G}^{0}(T M) \rightarrow C_{G}^{0}(T M) ; X \mapsto T f \cdot X \cdot f^{-1},
$$

is hyperbolic.

As an easy generalisation of the proof for $G=\mathrm{id}$, using Corollary 1.1, we have:

TheOREM 6. G-Anosov maps are (equivariantly) structurally stable.

If $X \in C_{G}^{r}(T M)$ is s.t.:

1. $\Omega(X), \bmod G$, is finite.

2. $\Omega(X)$ consists of families of closed orbits and fixed points.

3. $X$ is 3-generic.

We say $X$ is a "G-Morse-Smale vector field". We could weaken condition 3 to only require 1 and 2 genericity together with weak $G$ transversality of stable and unstable manifolds; we have, with this weaker definition:

TheOREM 7. Every compact $G$ manifold admits a G-Morse-Smale vector field.

With a suitable topology on $C_{G}^{r}(T M)$ and a suitable definition of $G$-structural stability which, for reasons of space, we will not reproduce here, one may reasonably ask: 
Problem. Are G-Morse-Smale systems structurally stable?

Suffice it to say that the main problem here appears to arise from the fact that in the $G$-orbit decomposition of $M$, into sets of the same type-see [5] - some of these sets are noncompact.

\section{REFERENCES}

1. S. Smale, Differentiable dynamical systems, Bull. Amer. Math. Soc. 73 (1967), 747-819. MR 37 \#3598.

2. A. G. Wasserman, Equivariant differential topology, Topology 8 (1969), 127-150.

3. R. S. Palais, Foundations of global non-linear analysis, Benjamin, New York, 1968.

4. C. C. Pugh, M. W. Hirsch and M. Shub, Invariant manifolds (to appear).

5. R. S. Palais, in A. Borel, "Seminar on transformation groups," Ann. of Math. Studies, no. 46, Princeton Univ. Press, Princeton, N. J., 1960. MR 22 \#7129.

Warwick University, Coventry, England 